\title{
Progress and Prospect of Research on Autonomous Vehicle
}

\section{Pan Yuyan ${ }^{1}$, Shi Jianjun ${ }^{1,2}$, Shi Zeyu ${ }^{1}$}

${ }^{1}$ City Traffic of College, Beijing University of Technology, City Transportation Institute, Beijing, China

${ }^{2}$ Key Laboratory of Transportation Engineering, Beijing University of Technology, City Transportation Institute, Beijing, China

\section{Email address:}

panyuyan8879@sina.com (Pan Yuyan)

\section{To cite this article:}

Pan Yuyan, Shi Jianjun, Shi Zeyu. Progress and Prospect of Research on Autonomous Vehicle. Science Discovery.

Vol. 5, No. 5, 2017, pp. 385-389. doi: 10.11648/j.sd.20170505.24

Received: July 29, 2017; Accepted: September 9, 2017; Published: September 14, 2017

Abstract: The paper system combs the related concepts of autonomous vehicles, and describes the demarcation criteria of automatic driving vehicles. Summed up the status quo at home and abroad research, analyzes the existing problems and research methods. And its future development trend is forecasted.

Keywords: Autonomous Vehicles, Laws and Regulations, Development Prospects

\section{自动驾驶车辆研究进展及其展望}

潘芋燕 $^{1}$, 石建军 ${ }^{1,2}$, 师泽宇 ${ }^{1}$

1城市交通学院, 北京工业大学, 北京, 中国

2 北京市交通工程重点实验室, 北京, 中国

邮箱

panyuyan8879@sina.com（潘芋燕）

摘要: 文中系统梳理了自动驾驶车辆的相关概念, 阐述了自动驾驶车辆行为划分标准, 总结了国内外研究现状, 分析 了目前存在的问题和研究方法, 并对其未来发展趋势进行了展望。

关键词：自动驾驶车辆, 法律法规, 发展展望

\section{1. 引言}

技术的发展会影响交通出行的方式, 自动驾驶车辆技 术的飞跃发展为当前的许多交通问题提供了独特的解决 方案。自动驾驶车辆具有安全性、高效性、舒适性、环保 性等众多优点, 一旦突破技术瓶颈、法律法规等问题, 自 动驾驶车辆将会突飞猛进地发展并广泛应用于我们的日 常生活中。

据NHTSA估计, 人为错误占所有交通事故原因的 $90 \%$, 自动驾驶车辆的应用将会消除车祸中的人为失误, 并且车
辆可以根据自身优势, 降低堵车时间, 从而有效利用环境 和道路资源 ${ }^{1}$, 对环社会发展具有重要意义。

技术的完备、法规的健全、市场准入准则的完善、辅 助设施的健全都是发展自动驾驶车辆市场化的前提。据有 关研究显示, 预计自动驾驶车辆运营能力将可以减少 $400 \%$ 的车辆交通压力 $[1]$, 可想而知, 一旦自动驾驶商业化普及 应用, 将会极大地缓解城市交通拥堵压力, 降低人们的出 行成本, 为城市节省更多的公共空间。更有研究表明, 随 着智能网络的建设和驾驶环境的逐渐完善, 预计到 2025 年将迎来自动驾驶车辆的商业应用[2]。 


\section{2. 自动驾驶车辆基本知识}

\section{1 . 自动驾驶车辆相关概念}

RV（regular vehicles）普通车辆, 是美国高速公路安 全协会于 2013 年给出的定义, 指的是 0 级自动化的车辆, 即完全没有自动化的车辆, 在行驶过程中全权由人类驾驶 员操作。

AV (autonomous vehicles) 自动驾驶车辆, 是指车辆 自己可以控制行驶速度的汽车, 与 $R V$ 相比, 车辆间距可 以更小, 行驶速度更均匀, 减少车辆行驶过程中加减速的 次数。即自动驾驶车辆是一个集环境感知、规划决策、控 制行动等多项功能于一体的综合智能系统, 应用的环境有 地面、空中、水下、水面等。目前大部分自动驾驶车辆是 指地面自动驾驶车辆。

SAV（shared autonomous vehicles）共享自动驾驶车 辆, 是自动驾驶车辆的一种拥有权的形式, 本质上是自动 驾驶车辆的“出租车行为”, 即SAV将每个出行者从起点送 到终点, 并把车辆停放在乘客终点处或者继续前往下一个
顾客的始发地 $[3]$ 。使用 SAV 可以减少道路上车辆总数, 出 行拥堵将会缓解。

PAV（personal autonomous vehicles）私人自动驾驶车 辆, 相对共享自动驾驶车辆而言是拥有权属于私人的一种 自动驾驶车辆。购买、转卖或者用于通行行为都只受私人 支配, 车辆本身性能与其它自动驾驶车辆完全一样, 可以 实现车辆安全高效地自主驾驶, 为出行者提供舒适、便捷 的服务。

等级划分: 共分为 5 级。 0 级由驾驶员驾驶; 1 级具备 1 种以上自动化控制功能（如车道保持系统等）；2级以汽 车为主体执行多种操作功能; 3 级即当以车辆为主体的驾 驶行不通时可指示驾驶员切换为手动驾驶; 4级完全可以 无人驾驶。

\section{2. 自动驾驶车辆行为划分}

根据自动驾驶车辆智能行为的难易程度及其执行过 程中要交互的环境对象, 将自动驾驶车辆智能行为分为: 基本车辆控制行为、基本行车行为、基本交通行为、高级 行车行为、高级交通行为 $[4]$ 。具体如下表1所示。

表1 自动驾驶车辆智能行为划分。

\begin{tabular}{|c|c|}
\hline 典型行为 & 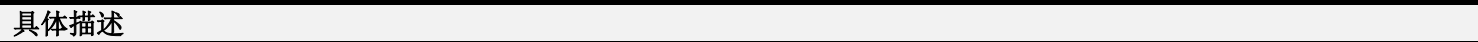 \\
\hline 基本车辆控制行为 & $\begin{array}{l}\text { 指自动驾驶车辆接收外界控制指令实现车辆启动、减速、停车以及停止操纵控制行为, 是自动驾驶车辆实现其他智能行为 } \\
\text { 的基础。 }\end{array}$ \\
\hline 基本行车行 & 驶车辆能够在没有其他动态车辆和人员等参与的简单道路交通环境中，遵守交通规驶等行为。沿着道路行驶，包括车道 \\
\hline 基本交通行为 & $\begin{array}{l}\text { 他动态车辆和人员等参与的简单道路交通环境中, 遵守交通规则, 正确地与其他动态交通参与车 } \\
\text { 行车任务, 包括跟驰行为、交叉口通行等行为。 }\end{array}$ \\
\hline 高级行车行为 & $\begin{array}{l}\text { 动态车辆和人员等参与的交通环境中完成的复杂行车任务, 如在低能见度环境下行驶、 } \\
\text { 线等行为。 }\end{array}$ \\
\hline 高级交通 & 耐, 正确地与其他动态 \\
\hline
\end{tabular}

\section{3. 国内外自动驾驶车辆发展现状}

目前自动驾驶发展研究主要是传统车企和互联网公 司对车辆的研发。2017年4月份，市场调研机构Navigant Research公布的全球自动驾驶全球实力排名显示, 传统车 企获得了压倒性的优势。具体如下表2所示。

表2 全球自动驾驶公司全球实力排名。

\begin{tabular}{ll}
\hline 排名 & 公司 \\
\hline 1 & 福特 \\
2 & 通用 \\
3 & Alliance \\
4 & 戴姆勒 \\
5 & 大众集团 \\
6 & 宝马 \\
7 & Waymo \\
8 & 沃尔沃 \\
9 & 德尔福 \\
10 & 现代 \\
\hline
\end{tabular}

2017年6月13日, 美国科技媒体The Information通过采 访和调研对当今世界主要的 17 家发展L4级别及以上无人 驾驶技术的公司进行了一次综合评估和排名, 主要从技术、
工程和商业模式三个指标评分，与市场调研机构Navigant Research公布的结果变化很大, 传统汽车制造商不具优势, 而互联网科技公司排名在前。具体如下表3所示。

表3 自动驾驶公司技术评估结果对比。

\begin{tabular}{llllll}
\hline $\begin{array}{l}\text { 综合 } \\
\text { 排名 }\end{array}$ & 公司 & 技术 & 工程 & 商业模式 & 评分 (满分 15) \\
\hline 1 & Waymo (谷歌) & 5 & 4.5 & 3.5 & 13 \\
2 & Uber & 3 & 4 & 5 & 12 \\
3 & 戴姆勒 & 3 & 3.5 & 3.5 & 10 \\
3 & 德尔福 & 3 & 3 & 4 & 10 \\
5 & 特斯拉 & 3.5 & 3 & 3 & 9.5 \\
6 & NuTonomy & 3 & 3 & 3 & 9 \\
7 & 百度 & 2 & 3 & 3.5 & 8.5 \\
8 & Cruise (通用) & 2.5 & 2.5 & 3 & 8 \\
8 & Renault (日产) & 2.5 & 2.5 & 3 & 8 \\
10 & 丰田 & 1.5 & 3 & 3 & 7.5 \\
10 & Argo (福特) & 2 & 2.5 & 3 & 7.5 \\
12 & 宝马 & 1.5 & 2.5 & 3 & 7 \\
13 & 奥迪 & 1.5 & 2 & 3 & 6.5 \\
14 & 沃尔沃+Autoliv & 1.5 & 1.5 & 3 & 6 \\
15 & 菲亚特克拉斯勒 & 1.5 & 2 & 2 & 5.5 \\
15 & 苹果 & 1.5 & 3 & 1 & 5.5 \\
17 & 滴滴出行 & 0 & 0.5 & 4.5 & 5 \\
\hline
\end{tabular}


如下表4所示, 根据各国政府、汽车公司或互联网企 业的规划, 2020年是自动驾驶产业发展的关键节点。美国、 德国、日本、中国等诸多企业都将2020年定为实现自动驾 驶的实现年, 并且技术竞争研发越来越激烈。

表4 各国或企业发展自动驾驶车辆计划表。

\begin{tabular}{ll}
\hline 国家或企业 & 发展计划 \\
\hline 美国谷歌 & 致力于2020年之前实现完全自动驾驶 \\
美国Lyft & 预计在2021-2022年实现所有服务由全自动驾驶车 \\
& 辆完成 \\
美国福特 & 计划在2021年之前为出租车或其他拼车服务公司 \\
& 提供自动驾驶车辆 \\
美国Uber & 计划在2020你那向公众展示它的第一款飞行出租 \\
美国特斯拉 & 车服务 \\
德国大众 & 到2020年实现新售汽车全部搭载自动驾驶技术 \\
& 将在2020年前后在每个细分市场都推出全自动驾 \\
德国戴姆勒 & 驶汽车 \\
德国宝马 & 计划在2020年之前开始销售自动驾驶车, 2020卡车全面部署 \\
韩国现代 & 计划在2021年于中国市场推出自动驾驶汽车 \\
韩国Naver & 预计2020年3级自动驾驶汽车将上市销售 \\
& 计划于2020年前商业化3级自动驾驶汽车 \\
日本Softbank & 计划于2020年将研发的一款自动驾驶公交车投入 \\
日本东京 & 商用 \\
& 计划于2020东京奥运会之际, 实现在高速公路上自 \\
中国百度 & 动驾驶的目标 \\
\hline
\end{tabular}

2012年5月, 谷歌自动驾驶汽车正式获得美国内华达 州车辆管理局 (DWV) 颁发的执照, 这是美国历史上为 自动驾驶车辆颁发的第一张执照。尽管谷歌自动驾驶车辆 上路行驶里程已超过 300 万英里, 但谷歌自动驾驶车辆已 经发生过25次交通事故, 但仅有一起事故是由于车辆本身 的问题所引发。

2016年9月份Uber首次推出无人驾驶汽车载客服务, 在美国匹兹堡上路试运行。随后2017年在旧金山进行第二 次试运行, 但Uber的自动驾驶车辆一直处于车内技术人员 的监控之下, 自动驾驶车辆随时可以被人工接管。Uber 目前正在开发软件控制系统, 在不久的将来将实现乘客利 用手机呼叫自动驾驶汽车, 出租车司机在这个古老的行业 里即将面临被淘汰的命运[5]。

特斯拉自动驾驶汽车并不是完全的自动驾驶, 是处于 L2级别的自动驾驶。首起自动驾驶致死的事故发生在中国, 车主开启了特斯拉自动驾驶模式, 结果酿成了惨剧。2017 年7月18日, 特斯拉自动驾驶再出现车祸, 突然加速导致 翻车, 并且 5 人受伤。

百度公司于 2015 年正式对外发布无人驾驶汽车, 并在 今年4月份宣布推出“阿波罗” (Apollo) 项目, 开放自动驾 驶平台。2017年7月5日百度自动驾驶车辆实现在北京五环 上路行驶, 该车辆没有配备激光雷达和摄像头, 仅依赖简 单的定位系统来实现固定线路上的自动驾驶。

\section{4. 发展自动驾驶车辆需要解决的问题}

\section{1. 技术瓶颈}

自动驾驶车辆目前面临两大难题。其一是车辆系统必 须有能够正确处理复杂多变的城市交通状况的能力, 因此 城市交通是研发该系统的难题之一。这一难题是在自动驾 驶车辆发展变革时期面临的重大瓶颈。需要对自动驾驶车 辆的软硬件的技术提高, 对传感器、GPS技术、雷达摄像、 $\mathrm{V} 2 \mathrm{X}$ 等都提出新的挑战, 尤其是 $\mathrm{AI}$ 算法, 是支撑自动驾驶 技术的最关键的部分 [6]。通过算法将收集的数据及驾驶行 为、规则、案例和周边环境的数据信息进行处理, 并不断 优算法, 使其能够识别规划路径、操纵驾驶。

其二是自动驾驶系统必须有预测判断的能力。自动驾 驶车辆要有预测目前行驶的车辆是否会在前方停车的能 力以及目前目标车道上的前方几百米是否出现拥堵现象 等。这些状况都需要自动驾驶车辆与中央控制指挥系统实 现信息交互, 需要指挥中心实时给自动驾驶车辆发布信息, 这一难题是在自动驾驶车辆普及时期面临的重大问题。

\section{2. 法律法规}

2016年9月21日, 美国交通运输部(DOT)与国家公路 交通安全管理局(NHTSA)联合颁布《联邦自动驾驶汽车 政策》, 成为全球首个由国家发布的自动驾驶规范性纲 领，为自动驾驶技术提供了制度保障[7]。欧盟及日本将 为自动驾驶车辆制定统一的法规标准, 将于2018年生效, 这一法规将会加快自动驾驶汽车的量产和商业化步伐。 尽管关于自动驾驶车辆的法规在不断完善, 但目前主要 面临三大难题: 市场准入准则、事故责任认定赔偿、安 全隐私保护。

安全性评估是自动驾驶车辆合法上路行驶的前提条 件, 是自动驾驶车辆法规的重要组成部分。一辆自身生产 合格的自动驾驶车辆不但要实现保护车内人员安全, 还要 实现如何避免与车辆外行人、车辆发生碰撞, 这就需要上 路测试, 在真实的环境中测试自动驾驶车辆是否具有处理 复杂情况的能力。目前国际和国内还未出台统一的测试标 准，对于政府来说，如何制定出一个客观、可测试多种可 能发生路况的自动驾驶车辆测试标准是一个非常艰巨的 挑战, 需要从环境感知、控制执行、功能安全、信息安全、 软件成熟度、行为决策、路径规划和综合智能等方面提出 一种系统的、全面的自动驾驶车辆测评方法, 完善市场准 入准则, 是目前面临的难题。

目前谷歌、Uber、特斯拉自动驾驶车辆都发生过交通 事故, 甚至造成人员伤亡, 已经引起公众普遍关注。另一 难题是自动驾驶车辆事故认定赔偿问题。自动驾驶车辆自 身是车辆的控制主体, 如果发生事故, 应由车辆的生产者 还是车辆的使用者来承担事故责任是法规必须回答的问 题。现有的责任案例法规没有提出对车主或驾驶员有特别 的责任考虑, 自动驾驶责任事故完全有别于传统操作的车 辆, 因此, 对事故的认定赔偿问题, 是一个十分棘手的问 题。

除了安全性评估和事故责任问题以外, 自动驾驶车辆 法规还涉及个人隐私的问题。为了保证车辆的行驶安全性, 
谷歌和各大汽车厂商通常都会对车辆行驶状态信息进行 详细的记录, 以监测车辆的正常行驶。被记录的车辆行驶 状态信息不仅包括车辆的车速、行驶里程, 而且包括车辆 的行驶位置及轨迹, 而车辆的行驶轨迹信息涉及驾驶人、 乘坐者的个人隐私。如何在保证车辆行驶安全的情况下记 录其行驶状态信息, 同时又不侵犯个人隐私也是自动驾驶 法规要回答的问题。

\section{3. 人类接受度}

自动驾驶车辆的一个标志性特征就是信息化程度极 高, 这对电脑系统的安全问题构成极大的挑战。为了更好 地发挥自动驾驶车辆的优势, 实现安全、高效地行驶, 自 动驾驶车辆通过“车车通信”和“车路通信”来实现车辆之 间的信息互通和信息共享。然而这些信息包括车辆的行驶 轨迹以及使用者的个人出行信息, 一旦电脑信息系统的安 全遭到破坏，势必导致使用者的信息泄露。这对于使用者 来说, 并不容易突破心理防线, 就目前调查来看, 人们对 自动驾驶车辆整体仍然存在犹豫观望态度, 有 $44 \%$ 的人仍 然选择使用常规车辆, 而即使自动驾驶车辆使用成本免费, 仍然会有 $25 \%$ 的人表示坚决拒绝使用自动驾驶车辆 $[8]$ 。因 此, 自动驾驶车辆的发展需要面对如何提高人类接受度的 问题，使其普遍被大众所接受。

\section{5. 自动驾驶车辆研究方法}

\section{1. 仿真模拟法}

目前对自动驾驶车辆的仿真研究分为对自动驾驶车 辆的仿真研究 [9]和对自动驾驶车辆运营环境的仿真研究 $[10]$ 。

自动驾驶车辆性能 (如定位技术、车体控制、安全) 的好坏、运行过程中对道路环境以及其他车辆和行人有 何影响, 都是需要测试的首要问题。而仿真模拟方法依 靠其低成本、可重复、多选择等优势成为解决此问题的 主要手段。目前普遍采用的研究思路是改变仿真软件中 的车辆模型的参数, 构造智能体使其具有自动驾驶车辆 的某些属性, 然后在虚拟的路网环境中观测车辆的运行 状况。自动驾驶系统是自动驾驶车辆运营所依赖的环境, 对自动驾驶车辆的运营环境进行仿真并使其最真实地逼 近真实环境, 是提高自动驾驶车辆测试水平的一个关键 问题。

\section{2. 真车实验法}

实验法即是对自动驾驶车辆在真实的环境中测试, 可 由动态模拟实验室、半封闭道路测试环境、全开放道路测 试环境三大测试部分组成。

其中动态模拟实验室是在实验室环境中，通过电脑 安装程序, 运用虚拟现实技术模拟真实的道路环境, 使 得驾驶员沉浸在虚拟的自动驾驶场景中, 从而对自动驾 驶车辆进行研究。半封闭道路测试环境不仅包括主干道、 交叉口、智能停车场、交通信号灯等道路场景, 还能模 拟雨、雪、雾等各种自然环境, 与真实环境有所差异,
并不能够达到真实交通运行环境的复杂程度。全开放道 路测试环境是在真实的路网环境中实现，例如谷歌、特 斯拉等开发的自动驾驶车辆最终都要经过真实道路的测 试, 才能对自动驾驶车辆做出最真实的技术、安全、运 行等方面的评价。

\section{6. 自动驾驶车辆发展展望}

\section{1. 自动驾驶车辆的产业发展}

目前研究自动驾驶车辆主要是互联网科技公司和传 统汽车企业。随着自动驾驶技术的完善以及高科技的发展, 信息通信行业和传统汽车产业融合的趋势会越来越明显 [11]。互联网公司具有信息通信高科技的优势，而传统汽 车企业具有车辆制造的优势, 如果二者融合起来, 势必给 自动驾驶车辆产业发展带来飞跃的发展。如何使得二者深 度融合并发挥各自的优势, 将会是未来发展的方向和值得 探讨的问题, “互联网+传统车企”必将是自动驾驶车辆产 业发展的新方向。

\section{2. 自动驾驶车辆的应用发展}

未来预计自动驾驶车辆发展将由规范运营试点示范 区到封闭区域再到开放式道路全面推广, 进一步向前演进, 城市道路公共交通有望实现自动驾驶, 城市出租车有望实 现按需供给的自动驾驶, 形成自动驾驶的城市公共汽车、 出租汽车和普通车在道路上混合行驶。最终, 大部分道路 将由自动驾驶车辆独占路权, 普通车辆在大部分道路上将 不允许上路行驶。路网上实现汽车使用率最大化, 个人使 用成本最低。具体应用过程如下:

单个/编组自动驾驶的应用会首先在示范区高速公 路上实现，并逐步应用到城市公交专用道。而一旦自动 驾驶车辆突破技术瓶颈, 度假村、港口、旅游景区、货 场、矿区、机场等封闭的网络地区的车辆将会首先被自 动驾驶车辆替代, 无人化港区、货场等将会成为现实, 景区的摆渡车辆将无需人工操作, 配置有道路电子信标、 路测传感装置的自动驾驶车道将会在局部地区全面部署, 最后实现在完全开放的道路全面普及应用自动驾驶车辆, 这一阶段的实现比较困难, 需要各种技术的集合应用, 并且还需要法律法规、责任事故划分、赔偿保险等问题 的健全和完善。

\section{7. 结论}

自动驾驶是科技发展的方向, 这个方向是不可逆的。 虽然发生的几起事故令人惋惜, 但是从总体来说, 自动驾 驶汽车导致的事故的概率非常低。当前自动驾驶技术发展 还很不完善, 需要突破众多技术瓶颈, 并且法律法规也相 对没有出台适合自动驾驶发展的相关策略, 政府应更科学、 专业的引领制定一个测试、评判的标准, 来明确自动驾驶 技术的发展路线，最大限度地解决和规避自动驾驶面临的 困境。 


\section{参考文献}

[1] Soyong Ahn.Towards vehicle automation:Roadway capacity formulation for traffic mixed with regular and automated vehicles [J]. Transportation Research Part B (100) 2017:197-221.

[2] Michael W. Levin. A general framework for modeling shared autonomous vehicles with dynamic network-loading and dynamic ride-sharing application [J]. Computers, Environment and Urban System, 2017.(06), 64:373-383.

[3] Daniel J. Fegnant. The travel and environment implications of shared autonomous vehicles, using agent-based model scenarios [J]. Transportation Research Part C :Emerging Technologies, 2014. (03), 40:1-13.

[4] 熊光明. 无人驾驶车辆智能行为及其测试与评价 $[M]$. 北京: 北京理工大学出版社, 2015.(11): 91-92。

[5] 阮晓东. Uber开测无人驾驶汽车, 意欲何为? [J]. 新经济 导刊, 2016.(10):54-59。

[6] 伦一. 自动驾驶产业发展现状及趋势 $[\mathrm{J}]$. 电信网技术, 2017.(06):33-36。

[7] 伦一. 美国自动驾驶立法政策的启示 $[\mathrm{J}]$. 互联网天地, 2016.(12):20-24。

[8] Chana J. Haboucha. User perferences regarding autonomous vehicles [J]. Transportation Research Part C, 2017. (04):37-49.

[9] 石坚, 卓斌. 自动驾驶汽车的仿真 $[\mathrm{J}]$. 汽车工 程. 2000.(02):97-100。

[10] 宋振伟. 基于FPGA的车辆自动驾驶系统的研究与仿真设计 [D]. 哈尔滨: 哈尔滨理工大学, 2014。

[11] 陈晓博. 发展自动驾驶汽车的挑战和前景展望 $[\mathrm{J}]$. 综合运输, 2016.(11):9-13。 